\title{
Gráfico de controle da média móvel exponencialmente ponderada aplicado ao monitoramento estatístico de um processo de usinagem
}

\author{
Vinicius Moretti (UNIVILLE-SC) vinimoretti20@hotmail.com \\ Giovani Paglia DegImann (UNIVILLE-SC) giovani.paglia14@gmail.com \\ Custodio da Cunha Alves (UNIVILLE-SC) custodio.alves@univille.br \\ Josiane Costa Riani (UNIVILLE-SC) Josiane.riani@univille.br \\ Gilson João dos Santos (UNIVILLE-SC) gilson.joao@univille.br
}

Resumo: O monitoramento de pequenas e permanentes alterações nos parâmetros da média de um processo é uma tarefa efetivamente importante, numa perspectiva de otimizar a estabilidade da qualidade de processos e produtos. Os gráficos de controle da média móvel exponencialmente ponderada (EWMA) são ferramentas de controle estatístico de processos relevantes e de grande sensibilidade na rápida detecção de pequenas e moderadas mudanças, em contraste com os tradicionais gráficos de controle de Shewhart apropriados para grandes mudanças. Este artigo propõe a aplicação do gráfico EWMA para a média de subgrupos racionais no monitoramento estatístico de um processo de usinagem que apresenta uma variação de $1 \sigma$ no valor da média. Com efeito, verificouse que, o gráfico de Shewhart para os dados reais deste processo não apresentou qualquer situação de anormalidade para o valor da média, enquanto que a aplicação do gráfico EWMA permitiu, dentro do tempo previsto, tal deteç̧ão.

Palavras chave: Monitoramento de processos, Gráfico EWMA, Alterações da média.

\section{Exponentially weighted moving average control chart applied to statistical monitoring of a machining process}

\begin{abstract}
Monitoring small and permanent changes in process parameters is an effectively important task from the perspective of optimizing process and product quality stability. Exponentially Weighted Moving Average (EWMA) control chart are highly sensitive and relevant statistical process control tools for the rapid detection of small and moderate changes, in contrast to traditional Shewhart control charts suitable for large changes. This paper proposes the application of the EWMA chart for the average of rational subgroups in the statistical monitoring of a machining process that presents a $1 \sigma$ variation in the mean value. Indeed, it was found that the Shewhart chart for the actual data of this process showed no abnormality for the mean value, whereas the application of the EWMA chart allowed such detection within the predicted time.
\end{abstract}

Key-words: Process monitoring, EWMA chart, Changes in average.

\section{Introdução}

O controle estatístico de processos é, sem dúvida, uma poderosa metodologia desenvolvida para auxiliar no controle eficaz da qualidade. Envolve um conjunto de ferramentas de resolução de problemas apropriado para fornecer informações que nos permite atuar em um processo na obtenção da estabilidade e na melhoria da capacidade através da redução da variabilidade.

O monitoramento efetivo das características da qualidade de um processo de produção depende frequentemente destas ferramentas estatísticas para a detecção, a identificação e a análise das causas significantes responsáveis por variações que afetam o comportamento do processo de maneira imprevisível. Entre estas ferramentas, estão os gráficos de controle que permitem a redução sistemática da variabilidade nas características da qualidade do produto 
representadas pelas variáveis monitoradas nos gráficos em função da sequência de amostras coletadas ao longo do tempo.

Dentre todos os gráficos de controle, o tradicional gráfico do tipo Shewhart é de fácil implementação e é eficiente para detectar grandes alterações em um processo. No entanto, para pequenas ou moderadas alterações, não é tão eficiente em comparação com alguns gráficos do tipo com memória (ponderados pelo tempo), tais como os clássicos gráficos CUSUM e EWMA que podem ser aplicados com sucesso no monitoramento do desempenho de diferentes processos industriais. Estes gráficos podem complementar ou substituir com vantagens os tradicionais gráficos de Shewhart e permitir, em função do caso em análise, a obtenção de uma solução mais precisa, a um custo e prazos menores que os requeridos pelas metodologias tradicionais.

Os gráficos de controle CUSUM e EWMA são projetados de tal forma que sua estatística de plotagem utiliza tanto observações do passado quanto atual o que os torna mais sensíveis a pequenos e moderados desvios do valor nominal nos parâmetros de interesse do processo enquanto o gráfico do tipo Shewhart envolvendo a estatística de plotagem e regras de decisão adicionais tem como base apenas a última observação.

O gráfico do tipo Shewhart, permite determinar a existência de causas assinaláveis de variação, sempre que o valor da estatística amostral se encontre fora dos limites de controle. Uma outra situação que poderá ocorrer, é a alteração do valor de determinado parâmetro do processo, sem que tal mudança seja detectada no respectivo gráfico de controle. Sempre que se verifique esta situação, como por exemplo, a alteração da média do processo, vai existir um período mais ou menos longo, em que a produção será pouco consistente. Além disso, a aplicação da totalidade ou de parte das regras de decisão aumentam, por um lado, a sensibilidade desse gráfico, como pretendido, mas por outro, aumentam o risco de falsos alarmes, o que é definitivamente inaceitável.

Conforme literatura, uma variedade de investigações que envolvem aperfeiçoamentos e modificações para o controle eficaz dos parâmetros e a melhoria da qualidade das saídas do processo, têm sido propostos com o propósito de melhorar ainda mais o desempenho desses gráficos e, sobretudo aumentar as habilidades de detecção de diferentes tipos de gráficos de controle. Recentes trabalhos de pesquisa relacionados a projetos de gráficos de controle com memória envolvendo o aprimoramento e a aplicação desses gráficos no monitoramento de processos e, em particular o desenvolvimento do gráfico EWMA podem ser encontrados em Abbas, Riaz e Does (2011, 2014), Hawkins e Wu (2014), Castagliola e Figueiredo (2015), Nazir, Abbas, Riaz e Does (2016), Zwetsloot, Schoonhoven e Does (2016), Sanusi, Riaz, Adegoke e Xue (2017), Raji (2015), Raji, Abbas e Riaz (2018), Alves, Konrath, Henning, Paladini e Oliveira (2019), Aslam, Rao, Khan e Al-Abbasi (2019), e Sanusi, Mukherjee e Xie (2019).

O presente trabalho propõe o gráfico de controle EWMA para média de subgrupos racionais no monitoramento estatístico de um processo de usinagem que apresenta uma variação de $1 \sigma$ no valor da média. Neste estudo, o gráfico EWMA é aplicado aos dados reais desse processo como um procedimento de controle eficaz e uma alternativa ao tradicional gráfico de Shewhart para detectar moderadas mudanças e, em particular para mudanças de menor magnitude desse processo. O objetivo é investigar se há diferença significativa quanto a sensibilidade existente entre a aplicação desses gráficos e, sobretudo reduzir o tempo de detecção de pequenas mudanças, sem aumentar a taxa de alarmes falsos.

Este artigo está estruturado em quatro seções, incluindo a presente introdução. A seção 2 traz 
o referencial teórico envolvendo os conceitos e fundamentos do tradicional gráfico do tipo Shewhart e dos clássicos gráficos de controle com memória (padronizado pelo tempo) para auxiliar nas interpretações e análises inerentes a aplicação desses gráficos no monitoramento de processos; na seção 3 está a metodologia cuja sistemática de aplicação envolve o desenvolvimento dos gráficos de controle em ambiente MS-Excel ${ }^{\circledR}$ mediante a utilização de dados reais do processo de usinagem. Na sequência, os resultados são apresentados e discutidos; e, finalmente, a seção 4 apresenta as considerações finais.

\section{Referencial Teórico}

O Controle Estatístico de Processos (CEP) e em particular as técnicas de controle da qualidade, tais como gráficos de controle têm sido cada vez mais importantes pelo fato de desempenharem papel primordial na indústria moderna.

O maior objetivo do CEP é a eliminação da variabilidade e os gráficos de controle são ferramentas eficientes que permitem a redução sistemática dessa variabilidade nas características de qualidade do produto representadas pelas variáveis monitoradas no gráfico (MONTGOMERY, 2016; QIL, 2014). Neste contexto, o monitoramento contínuo do desempenho de um processo via CEP é uma atividade essencial para melhorar a qualidade de produtos e serviços (ABBAS \& SAEED, 2019).

A seleção adequada do tipo de gráfico de controle no monitoramento de processos é um ponto de partida vital para o CEP, uma vez que, a utilização de qualquer tipo de gráfico de controle depende principalmente, da classificação dos dados, do tipo de distribuição e da intenção da aplicação. Com isso, a seleção incorreta do tipo de gráfico pode resultar em muitos falsos alarmes e custos de monitoramento altos e inúteis para as causas especiais do processo (ALVES, et al., 2019).

Nesta seção, são concentrados inicialmente alguns fundamentos referentes ao tradicional gráfico de controle para variáveis desenvolvido por Shewhart como referencial teórico necessário ao entendimento e a aplicação dos conceitos e fundamentos que envolvem outras alternativas de gráficos de controle utilizados no monitoramento de processos para auxiliar nas interpretações e análises inerentes a esta técnica de controle de qualidade. Os clássicos gráficos com memória CUSUM e EWMA representam uma dessas alternativas. A aplicação do gráfico EWMA para a média de subgrupos racionais é proposta para o monitoramento estatístico de um processo de usinagem que apresenta uma variação de $1 \sigma$ no valor da média, objeto de estudo desse trabalho.

\subsection{Gráfico de Shewhart}

O modelo geral dos gráficos de controle para variáveis baseado nas premissas apresentadas por Shewhart abrange uma estatística de amostra $W$ que mede continuamente a variabilidade de algumas características de qualidade de interesse supondo a média dessa estatística $\mu_{w}$ com um desvio $\sigma_{w}$. Os valores desta estatística a serem plotados no gráfico de controle são obtidos a partir de cada uma das amostras coletadas do processo em períodos de tempo determinados pelos subgrupos racionais. Dessa forma, o modelo geral dos gráficos de controle para variáveis é definido a partir dos seguinte parâmetros

$$
L S C / L I C=\mu_{w} \pm k \sigma_{w} \quad \text { (Limites Superior e Inferior de Controle) }
$$

$$
L C=\mu_{w} \quad \text { (Linha Central) }
$$

onde $k$ é a distância da linha central a cada um dos limites de controle expressa em termos de 
unidades de desvio padrão.

Deste modo, se os valores da estatística de amostra $W$ caírem dentro dos limites de controle (LSC e LIC) o processo produtivo deverá prosseguir. Caso contrário, o processo deverá ser parado para identificar a causa determinística que provocou tal situação. Se adotarmos k=3 os parâmetros do modelo geral dos gráficos de controle ficarão tais que

$L S C / L I C=\mu_{w} \pm 3 \sigma_{w} \quad$ (Limites Superior e Inferior de Controle)

$L C=\mu_{w}$

(Linha Central)

Historicamente, a adoção do valor $k=3$ para o cálculo dos limites de controle $3 \sigma$ se tornou um padrão aceito na maioria dos processos industriais existentes.

Se os parâmetros média e desvio padrão do processo forem conhecidos, os limites de controle são atribuídos em $\mu \pm 3 \sigma$, contanto que seja razoável presumir uma distribuição normal como um modelo apropriado. Da mesma forma que nos métodos estatísticos em geral, os pressupostos devem ser checados. Tradicionalmente, o gráfico de controle $\bar{X}$ (subgrupos racionais) tem sido mais utilizado, e parece razoável presumir que ainda é mais empregado do que qualquer outro gráfico de controle (RYAN,2011).

Uma vez que os limites $3 \sigma$ são empregados tipicamente num gráfico $\bar{X}$, a distribuição de $\bar{X}$ deve ser razoavelmente simétrica e próxima de uma distribuição normal. Uma consideração importante sobre a distribuição dos dados no desenvolvimento do gráfico $\bar{X}$ segundo Ryan (2011) é que o número de subgrupos utilizado deverá proporcionar no mínimo 100 observações, ou seja, se temos subgrupos de tamanho igual a 5, precisamos de pelo menos 20 subgupos.

Seja $\bar{X}$ o valor de uma variável aleatória para qualquer item particular ou espécime. Considerando a hipótese de que para um processo sob controle $\bar{X}$ tem uma distribução normal com valor médio $\mu$ e desvio padrão $\sigma$. Então se $\bar{X}$ indica a média amostral para uma amostra de tamanho $\mathrm{n}$ selecionada em um instante de tempo particular, sabemos que $E(\bar{X})=\mu, \quad \sigma_{\bar{X}}=\sigma / \sqrt{n}$ e que $\bar{X}$ tem uma distribuição normal, então $P\left(\mu-3 \sigma_{\bar{X}} \leq \bar{X} \leq \mu+3 \sigma_{\bar{X}}\right)=P(-3 \leq Z \leq 3)=0,9974$ onde $Z$ é uma variável aleatória normal padronizada. Então é altamente provável que para um processo sob controle, a média amostral caia dentro dos $3 \sigma_{\bar{X}}$ da média do processo $\mu$.

Considere primeiro o caso no qual os valores de ambos $\mu$ e $\sigma$ são conhecidos. Suponha que em cada um dos pontos de tempo $1,2,3, \ldots$, uma amostra aleatória de tamanho $n$ esteja disponível. Seja $\bar{x}_{1}, \bar{x}_{2}, \bar{x}_{3}$, .. os valores calculados das médias amostrais correspondentes. 0 modelo do gráfico de controle do tipo Shewhart baseados em valores de parâmetros conhecidos (média $\mu$ e desvio padrão $\sigma$ ) frequentemente conhecido como gráfico de controle $3 \sigma$. Os limites de controle para esse gráfico é definido como

$L S C / L I C=\mu \pm 3 \frac{\sigma}{\sqrt{n}} \quad$ (Limites Superior e Inferior de Controle)

$L C=\mu$

(Linha Central)

Qualquer ponto fora dos limites de controle sugere que o mprocesso pode ter ficado fora de controle naquele momento, portanto, uma busca de causas assinaláveis deve ser iniciada.

É importante ressaltar que os valores dos parâmetros média, $\mu$ e desvio padrão, $\sigma$ de um 
processo geralmente são desconhecidos, portanto devem ser estimados a partir dos dados de amostra antes de determinar os limites de controle. Isto é especialmente verdadeiro quando um processo está sujeito à análise do controle de qualidade pela primeira vez.

Novamente, seja $n$ o número de observações em cada amostra e $k$ o número de amostras disponíveis. Considerando a hipótese de que as amostras $k$ foram coletadas durante um período quando se acreditou que o processo estava sob controle.

Com $\bar{x}_{1}, \bar{x}_{2}, \bar{x}_{3}, . ., \bar{x}_{k}$ indicando as médias amostrais $k$ calculadas, a estimativa usual de $\mu$ é simplesmente a média das médias

$\hat{\mu}=\overline{\bar{x}}=\frac{\sum_{i=1}^{k} \bar{x}_{i}}{k}$

Os limites de controle para um gráfico são calculados como

$\overline{\bar{X}} \pm 3 \hat{\sigma}_{\bar{x}}$

com $\overline{\bar{X}}$ representando a média das médias amostrais dos subgrupos e $\hat{\sigma}_{\bar{x}}$ representando algum estimador do desvio padrão das médias dos subgrupos. Esse estimador é obtido tipicamente como sendo $\hat{\sigma}_{\bar{x}}=\bar{S} / c_{4} \sqrt{n}$ com $\bar{S}$ representando a média dos desvios-padrão do subgrupo e $c_{4}$ o valor tabelado para um determinado tamanho de subgrupo, ou $\hat{\sigma}_{\bar{x}}=\bar{R} / d_{2} \sqrt{n}$ com $\bar{R}$ com sendo a média dos intervalos de subgrupos e $d_{2}$ o valor tabelado para um determinado tamanho de subgrupo.

\subsection{Gráficos com memória}

O gráficos de controle estatístico aplicado no monitoramento de processo mais conhecido e amplamente aplicado principalmente no setor industrial é ainda, sem dúvida, os tradicional gráficos de controle $\bar{X}$ de Shewhart. Apesar de eficaz, essa ferramenta estatística não é a única disponível para monitorar a qualidade de um processo. Em alguns casos outros tipos de gráfico de controle podem ser aplicados com a mesma finalidade e com vantagens. É o caso dos gráficos com memória tais como os clássicos gráficos CUSUM e EWMA.

O gráfico de controle $\bar{X}$ de Shewhart proporciona uma grande sensibilidade no diagnóstico de causas especiais (identificáveis) esporádicas ou intermitentes. No entanto, em situações em que há uma causa no sistema, que gera uma pequena e constante variação na média ou na variabilidade, esse gráfico apresentará uma tendência nos valores plotados para as amostras. Apesar de existirem regras práticas para executar este tipo de análise, detectar tal tendência nem sempre é fácil e exige uma certa prática por parte do responsável do processo. E, mesmo que venha a ser percebida, é difícil determinar por intermédio dos gráficos de Shewhart quando o processo começou a deteriorar-se. Exatamente nesses casos que a aplicação de gráficos com memória pode ser vantajosa, isto é, quando existe um outro tipo de causa especial persistente até que uma ação seja tomada para eliminar a causa (ALVES, et al., 2004).

Os gráficos com memória também conhecidos como gráficos ponderados pelo tempo acumulam a informação mais recente com informações anteriores em toda a sequência de pontos e, com isso, detectam pequenas e moderadas mudanças dos parâmetros de um processo com um número médio de amostras até o sinal ( $A R L$ ) bem menor do que fariam os gráficos de Shewhart que utilizam a informação sobre o processo contida apenas no último ponto demarcado. A ideia de gráficos com memória é que sua representação gráfica não se 
fundamenta em observações individuais, ou médias de subgrupos racionais, mas no acúmulo de observações ponderadas ao longo do tempo (LOUZADA et al., 2013).

Nas duas próximas seções são apresentados os dois clássicos gráficos de controle com memória CUSUM e EWMA mais utilizados como alternativa de maior sensibilidade para detectar com maior rapidez pequenas e moderadas mudanças dos parâmetros de um processo com observações independentes e normalmente distribuídas.

\subsection{Gráfico de controle de Soma Acumulada (CUSUM)}

O gráfico de controle de Soma Acumulada (CUSUM) clássico proposto por Page (1954) é uma alternativa ao gráfico do tipo Shewhart. Várias versões do gráfico CUSUM existem, mas a versão comumente utilizada é a Cusum Tabular (MONTGOMERY, 2016). No procedimento desta versão é utilizada a soma acumulada para acumular desvios de cada observação em relação ao valor nominal, $\mu_{\mathrm{o}}$ que estão acima do valor nominal com a estatística $C_{i}^{+}$e desvios de $\mu_{\mathrm{o}}$ que estão abaixo do valor nominal com a estatística $C_{i}^{-}$. As estatísticas $C_{i}^{+}$e $C_{i}^{-}$ denomindas CUSUM superior e inferior unilaterais, respectivamente, para monitorar a média de um processo e definidas por Hawkins e Olwell (1998), incialmente com $C_{0}^{+}=C_{0}^{-}=0$, como: $C_{i}^{+}=\operatorname{máx}\left(0, C_{i-1}^{+}+\bar{X}_{i}-\mu_{0}-K\right)$

$C_{i}^{-}=\min \left(0, C_{i-1}^{-}+\bar{X}_{i}-\mu_{0}+K\right)$

onde $\bar{X}_{i}(i=1,2, \ldots)$ são observações independentes e normalmente distribuidas, $\bar{X}_{i} \sim N\left(\mu_{0}, \sigma\right), \mu_{0}$ é o valor nominal e $\sigma$ o desvio padrão. O valor de referência, $K=k \sigma$ nas equações (9) e (10), é geralmente escolhido como a metade da magnitude de mudança (em unidades de desvios padrão), isto é, $K=\frac{\delta}{2} \sigma=\frac{\left|\mu_{1}-\mu_{0}\right|}{2}$. As quantidades $\mathrm{k}$ e $\mathrm{h}$ são parâmetros do gráfico CUSUM, e sua seleção adequada é muito importante uma vez que influencia muito no desempenho de ARL desse gráfico. Se $C_{i}^{+}$ou $C_{i}^{-}$, excede o intervalo de decisão, $H=h \sigma$ o processo é considerado fora de controle. Um valor razoável para $\mathrm{H}$ é cinco vezes o desvio padrão, $\sigma$ do processo, isto é, $H=5 \sigma$.

\subsection{Gráfico de controle de Média Móvel Exponencialmente Ponderada (EWMA)}

O gráfico de controle da Média Móvel Exponencialmente Ponderada (EWMA) proposto por Roberts (1959) é outra boa alternativa ao gráfico de controle do tipo Shewhart, se o objetivo é deslocar pequenos e moderados deslocamentos na média do processo (ALVES, et al.,2016). Esse gráfico acumula informações sucessivas ponderando as amostras atribuindo maior peso para as observações mais recentes e peso menor para as mais remotas, isto é, o peso dado às amostras decresce geometricamente da primeira até a última amostra. Seu desempenho em termos de ARL é bastante similar ao gráfico de controle CUSUM (RYAN,2011; QIL, 2014).

A estatística $Z_{i}$ do gráfico de controle EWMA para monitorar a média de um processo e definida incialmente como igual ao valor nominal $\left(Z_{0}=\mu_{0}\right)$ é determinada da seguinte forma $Z_{i}=\lambda \bar{X}_{i}+(1-\lambda) Z_{i-1}$

onde $\bar{X}_{i}(i=1,2, \ldots)$ são observações independentes e normalmente distribuidas, $\bar{X}_{i} \sim N\left(\mu_{0}, \sigma\right)$, $\mu_{0}$ é o valor nominal e $\sigma$ o desvio padrão. A constante de suavização $\lambda$ é um dos parâmetros do gráfico com valor entre $0<\lambda \leq 1$. Quando $\lambda=1$, o gráfico EWMA reduz-se ao gráfico do tipo Shewhart, assim como $\lambda=0, Z_{0}=\mu_{0}$. 
A estrutura de controle do gráfico EWMA que incluem o limite superior de controle (LSC), limite inferior de controle (LIC) e a linha central (LC) é definida como:

$$
\begin{aligned}
& L S C / L I C=\mu_{o} \pm L \frac{\sigma}{\sqrt{n}} \sqrt{\frac{\lambda}{2-\lambda}\left[1-(1-\lambda)^{2 i}\right]} \\
& L C=\mu_{0}
\end{aligned}
$$

onde o fator $L$ (equação 12 ) é a extensão dos limites de controle, ou seja, o número de múltiplos de desvio padrão em que os limites de controle estão distantes da linha central (LC). Assim como o gráfico CUSUM, o gráfico EWMA tem dois parâmetros, $\lambda$ determina a redução de pesos e $L$ a amplitude dos limites de controle e conjuntamente $(\lambda, L)$ determinam 0 desempenho ARL do gráfico EWMA.

Trabalhos de pesquisa que incluem várias modificações nos clássicos gráficos com memória CUSUM e EWMA foram desenvolvidas para aprimorar ainda mais o desempenho desses gráficos. Algumas dessas melhorias podem ser vistas em Lucas e Saccucci (1990), Hawkins e Olwell (1998), Steiner (1999), Castagliola et.al (2015), Machado e Costa (2008), Abbas, Riaz e Does (2011) e suas referências. Seguindo esses autores, Abbas et al.(2013) e Zaman et al. $(2014,2016)$ propuseram os gráficos de controle misto EWMA-CUSUM e CUSUM-EWMA, respectivamnente, e concluíram que a mistura dos dois gráficos CUSUM e EWMA torna cada um desses projetos de gráfico de controle misto proposto ainda mais sensível a pequenas mudanças na média do processo em comparação com os outros projetos desenvolvidos para situações semelhantes.

\subsection{Avaliação e Comparação de Gráficos de Controle}

A avaliação e a comparação de diferentes tipos de gráficos de controle são realizadas mediante a utilização de indicadores de desempenho estatísticos e econômicos. O desempenho estatístico de um gráfico de controle em termos de potência é geralmente mensurado através de parâmetros relacionados com a distribuição do tempo para esse gráfico emitir um sinal. O número médio de amostras até a emissão de um sinal (ARL) é um desses parâmetros que a um determinado nível de qualidade representa o número médio de amostras coletadas necessário para que seja detectada uma mudança no processo. Este sinal, pode ser tanto um alarme falso como um sinal de que o processo está fora de controle (ALVES \& SAMOHYL,2004; RIAZ, ABBAS \& DOES, 2011, NAZIR et al., 2015 ).

O projeto estatístico de um gráfico de controle envolve procedimentos que contemplem a otimização suficiente de seus parâmetros para maximizar a capacidade de deteç̧ão de mudanças reais deste gráfico. Do ponto de vista estatístico, um bom gráfico de controle é aquele que além de ter uma estrutura de projeto eficiente, contemple a importância mais prática que é ter resistência à situações inusitadas.

$\mathrm{Na}$ literatura, os gráficos de controle são geralmente projetados estatisticamante para minimizar o tempo de resposta do gráfico de controle, ou seja, o número médio de amostras até a emissão de um sinal (ARL), a uma transferência antecipada sob a taxa de falso alarme tolerável. No entanto, as mudanças num processo de várias fontes, muitas vezes vêm com diferentes tamanhos e resultam em diferentes graus de impacto na qualidade (CHEN, 2007).

O ARL é o indicador estatístico mais utilizado para avaliar e comparar o desempenho de gráficos de controle. Esse parâmetro leva em conta os valores dos erros Tipo I e Tipo II, isto é, o custo associado à procura do problema inexistente e o custo associado à fraca qualidade que se obtém no produto final desde quando a mudança ocorre até que seja detectada 
(ALVES, et al., 2013). Assim, para avaliar os parâmetros de um gráfico de controle, é recomendado analisar o comportamento de ARL diante das várias amplitudes de mudança uma vez que, o ideal é que o $A R L$ do gráfico seja grande quando o processo está sob controle $\left(A R L_{0}\right)$ e pequeno quando o processo está fora de controle $\left(A R L_{1}\right)$.

A figura 1 ilustra o desempenho em termos do número médio de amostras até a emissão de um sinal para um $A R L$ sob controle, $A R L_{0}=370$ dos gráficos do tipo $\operatorname{SHEWHART}(k=3)$, CUSUM $(k=0,5$ e $h=4,77)$ e EWMA $(\lambda=0,1$ e $k=2,7)$, segundo Costa, et al.(2005, p.193 e 197).

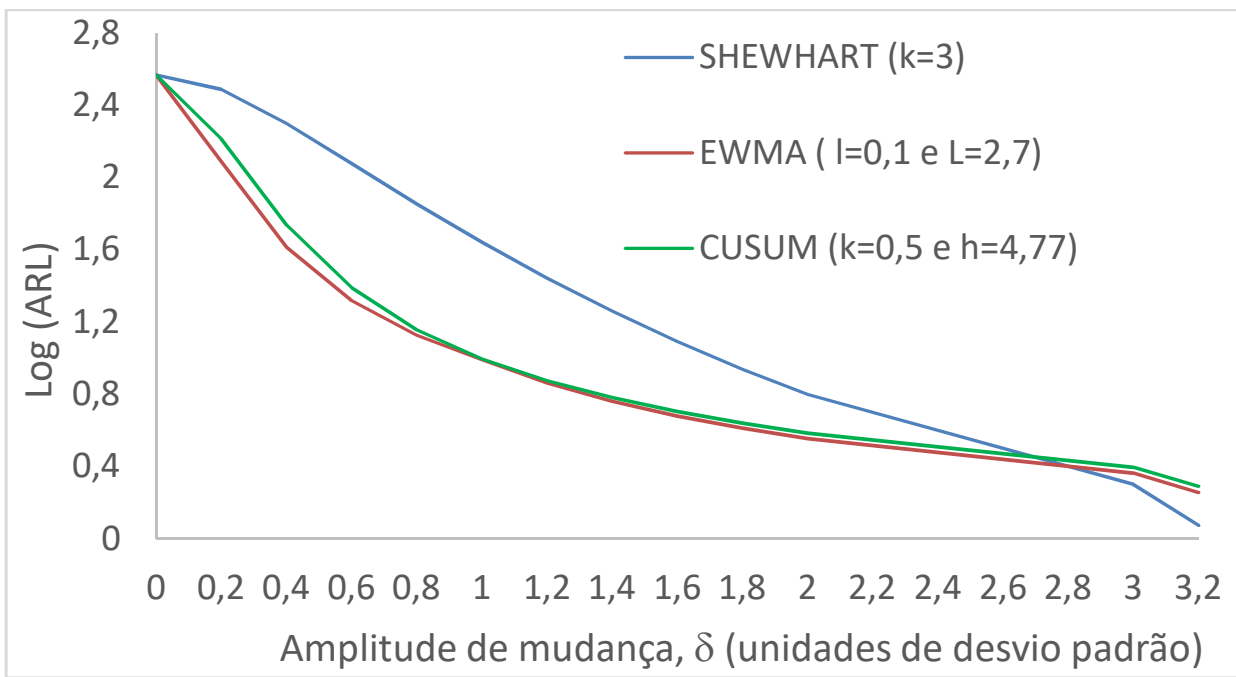

Figura 3 - Gráficos de controle do tipo SHEWHART, CUSUM e EWMA para os dados do processo

\section{Metodologia}

Este artigo além de apresentar as propriedades estatísticas e os procedimentos para ilustrar a lógica do processo de desenvolvimento dos três principais gráficos de controle até aqui apresentados, tem como objetivo mostrar como a metodologia de aplicação proposta para o projeto de cada um desses gráficos pode ser aplicada a uma situação real. Com esse propósito, o gráfico de controle EWMA para a média amostral de subgrupos racionais é aplicado nesse trabalho a um conjunto de dados no monitoramento de um processo de usinagem.

Os dados reais utilizados neste estudo são referentes a um processo de usinagem do corpo da válvula de descarga cedidos por uma indústria de metais sanitários localizada no norte do estado de Santa Catarina. Nesse processo a parte interna do corpo da válvula de descarga é usinada para obtenção de um alojamento no seu interior onde é inserido para vedar um anel oring de borracha durante a montagem da válvula de descarga.

O processo de usinagem desse alojamento para o anel oring requer uma alta precisão para evitar possíveis vazamentos durante sua utilização. A característica de qualidade utilizada para o monitoramento desse processo via gráfico de controle EWMA para a média amostral de subgrupos racionais é a medida diâmetro do alojamento para o anel de oring do corpo da válvula de descarga cujo valor nominal é $46,52 \pm 1,5 \mathrm{~mm}$, objeto de estudo desse trabalho. Esta característica de qualidade é atualmente monitorada através gráfico de controle $\bar{X}$ de Shewhart com 25 amostras de tamanho 4, coletadas diariamente a cada hora de produção.

Com base nestes em dados históricos e acompanhamento do processo a partir de vários conjuntos de dados recentes uma análise estatística do monitoramento desta característica de qualidade via gráficos de controle CUSUM e EWMA é ralizada para investigar o desempenho estatístico desses gráficos com memória. 
Ponta Grossa, PR, Brasil, 04 a 06 de dezembro de 2019

Um estudo preliminar da estrutura que inclui a organização de procedimentos e técnicas estatísticas para verificação da premissa e suposições de gráficos de controle paramétricos cuja modelagem estatística ou testes de hipóteses estatísticas, entre outros têm como pressuposto básico que os dados sejam distribuídos normalmente, independentes e identicamente distribuídos. Isso implica, em um procedimento eficiente para a verificação da premissa de que o processo encontra-se sob controle antes da implantação dos gráficos de controle e; das suposições de normalidade e independência. A verificação da premissa de estacionariedade e das suposições de normalidade, independência bem como a ausência de outliers do conjunto de dados do processo usinagem é também desenvolvida numa planilha eletrônica em ambiente MS-Excel ${ }^{\circledR}$ conforme figura 2.

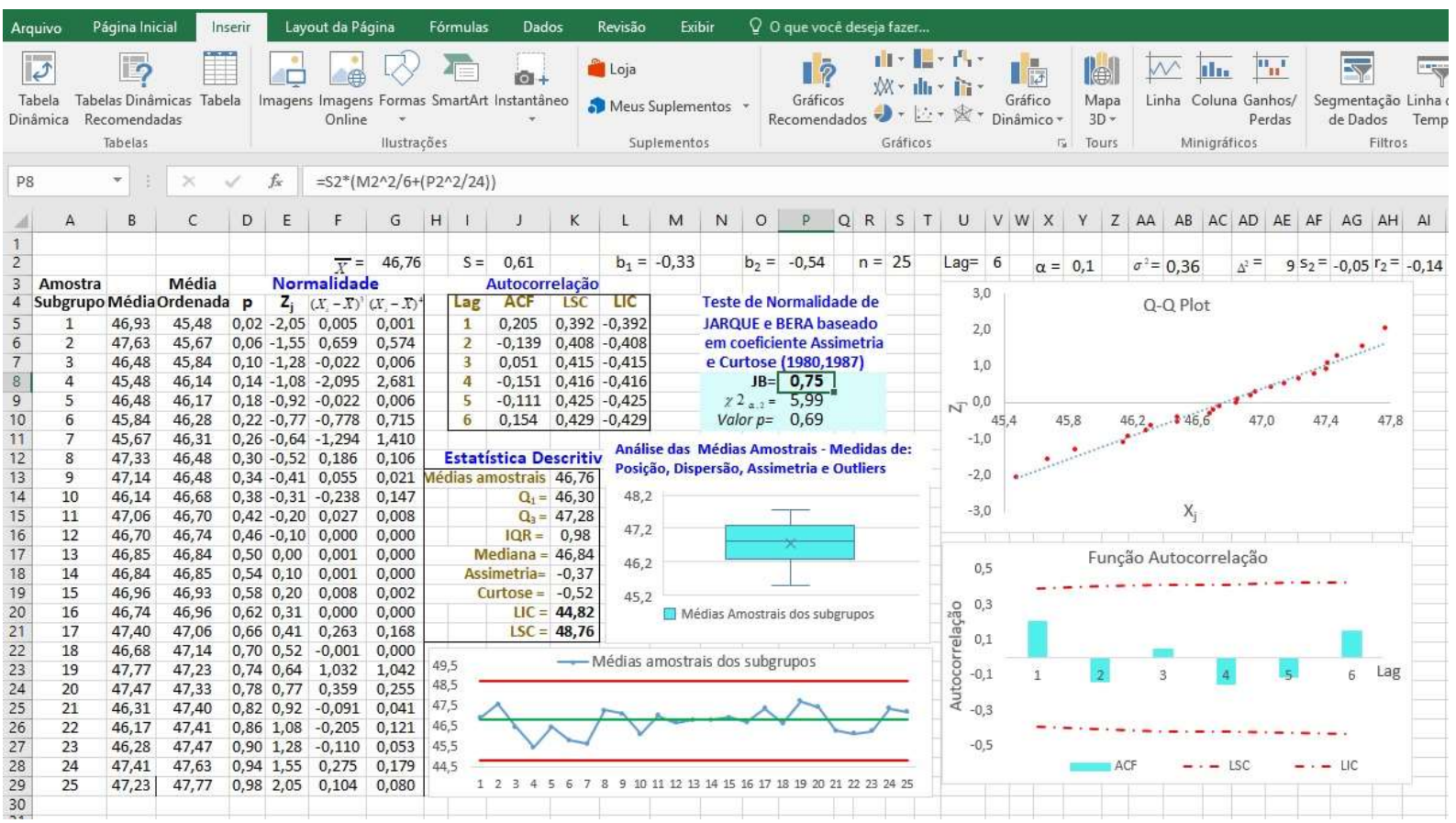

Figura 2 - Premissa e suposições normalidade, independência e ausência de outliers para os dados do processo

Os dados do processo de usinagem conforme ilustrado na figura 2, se comportam segundo uma distribuição normal ( $Q-Q$ Plot e Teste de Normalidade de Jarque e Bera), não apresentam autocorrelação (Correlograma) e ausência de outliers (Boxplot das médias amostrais dos subgrupos racionais e Estatística Descritiva).

A sistemática para o desenvolvimento dos três principais gráficos de controle do CEP e, em particular, o gráfico da Média Móvel Exponencialmente Ponderada, EWMA, envolve a aplicação de tais gráficos ao conjunto de dados do processo de usinagem, é desenvolvida numa planilha eletrônica em ambiente MS-Excel ${ }^{\circledR}$.

Para ilustrar a sitemática de modelagem no desenvolvimento do gráfico de Shewhart, optouse pela metodologia proposta por Weeler (1995). Para os gráficos com memória optou-se pelas metodologias propostas CUSUM (Hawkins e Olwell, 1998) e EWMA (Steiner 1999).No

Para o desenvolvimento dos três principais gráficos de controle e, em particular, o gráfico EWMA aplicado ao monitoramento do processo de usinagem, objeto de estudo deste trabalho, são utilizados parâmetros que tem como base o $A R L_{0}=370$ para um estudo comparativo do desempenho estatístico desses principais gráficos de controle, conforme figura 3. 


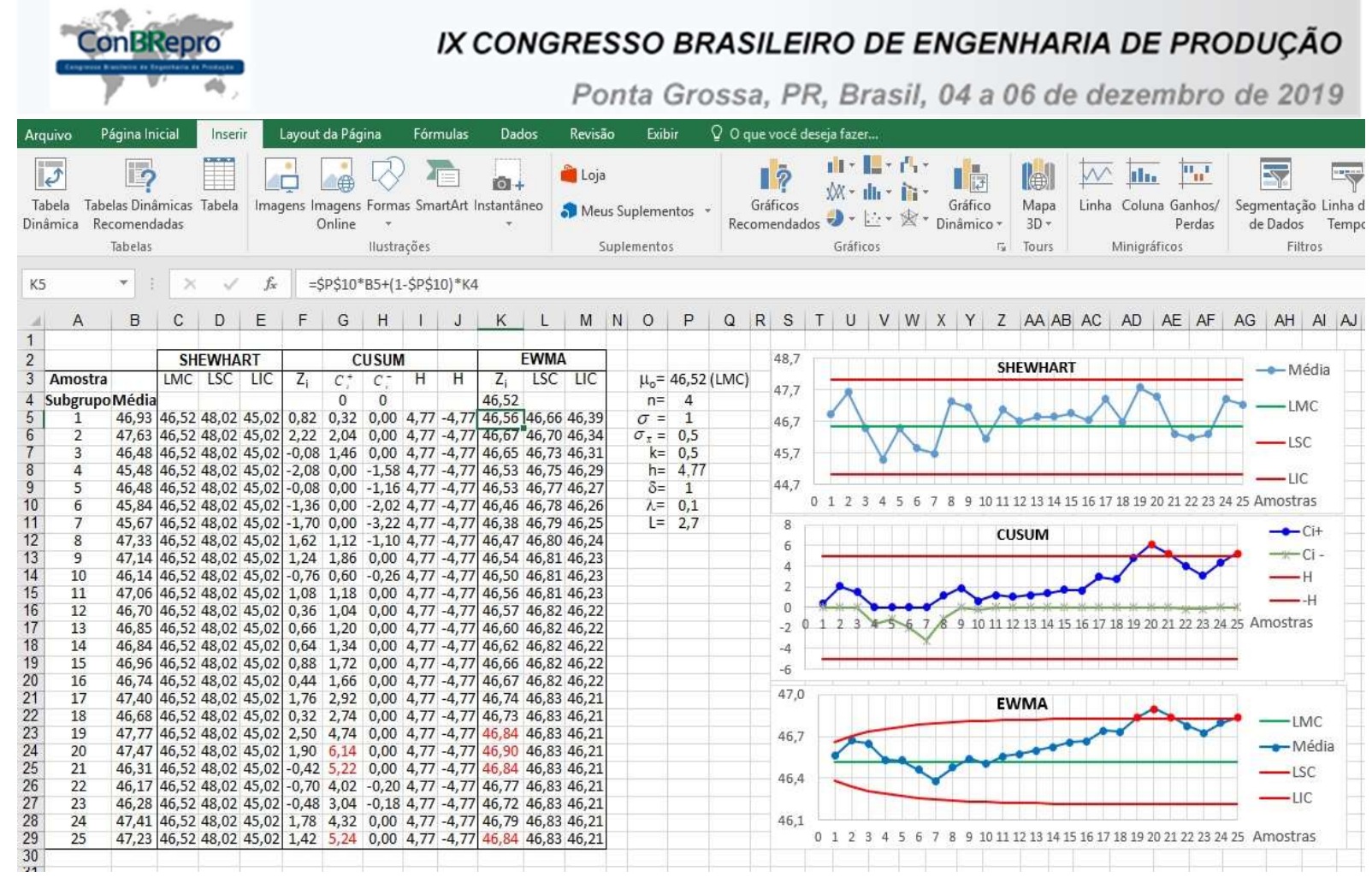

Figura 3 - Gráficos de controle do tipo SHEWHART, CUSUM e EWMA para os dados do processo

Os três principais gráficos de controle para os dados do processo de usinagem conforme ilustrado na figura 3, no gráfico $\bar{X}$ de Shewhart nenhum ponto ultrapassa os limites de controle. É possível melhorar a sensibilidade desse gráfico com o auxílio de regras suplementares, mas a incorporação dessas regras segundo Lousada et al. (2013), reduz a simplicidade de interpretação do gráfico e aumenta o número de falsos alarmes.

Os gráficos CUSUM e EWMA conforme figura 3 assinalam um deslocamento no nível médio do processo a partir da 11a amostra. O gráfico CUSUM sinalizou três pontos fora dos limites de controle a partir da 20a amostra enquanto o gráfico EWMA se apresentou um pouco mais sensível ao assinalar quatro pontos fora dos limites de controle já a partir da 19a amostra.

\subsection{Resultados e discussão}

Os resultados obtidos a partir de dados reais neste trabalho, mostram o gráficos com memória CUSUM e EWMA como ferramentas muito úteis no monitoramento desse processo de usinagem pois se mostraram mais sensíveis do que o tradicional gráfico de controle $\bar{X}$ de Shewhart para detectar a magnitude de mudança proposta de $1 \sigma$ para a média da característica de qualidade monitorada nesse processo. Tais resultados indicaram o gráfico EWMA com maior sensibilidade para o monitoramento desse processo uma vez que, permitiu dentro do tempo previsto, a detecção da magnitude de mudança proposta para a média da característica de qualidade desse processo.

Os resultados dessa aplicação comprovam o enunciado de que quanto menor for o ARL de um gráfico de controle para detectar num intervalo uma determinada magnitude de mudança, mais sensível será esse gráfico. Esses resultados comprovam esta premissa, como pode ser visualizado na figura 1, que ilustra o gráfico EWMA mais sensível que o gráfico CUSUM para o intervalo da magnitude de mudança, $\delta$ proposta que não ultrapassa a $1 \sigma$.

\section{Considerações finais}

Os modelos de gráficos de controle com memória, também conhecidos como gráficos de 
controle ponderados pelo tempo tais como os gráficos CUSUM e o EWMA são aprimoramentos dos gráficos do tipo Shewhart desenvolvidos para em situações específicas minimizar simultaneamente a ocorrência de alarmes falsos e alarmes não fornecidos.

Neste trabalho, o gráfico EWMA apesar de apresentar sensibilidade similar ao gráfico CUSUM para o conjunto de dados do processo de usinagem pode ser considerado mais sensível para detectar a magnitude de mudança proposta pois apresenta menor valor de ARL no intervalo da magnitude de mudança considerado. No entanto, de controle $\bar{X}$ de Shewhart não detectou qualquer situação fora de controle para o conjunto de dados fornecido. A análise estatística obtida a partir do estudo comparativo de desempenho relativo, em termos de ARL, revelou os clássicos gráficos memória significativamente mais sensíveis que o tradicional gráfico $\bar{X}$ de Shewhart para o conjunto de dados desse processo.

A estatística EWMA desse gráfico com memória apresenta múltiplas aplicações, ou seja, é aplicada não apenas como uma ferramenta estatística de monitoramento e ajustes de processos, mas, também como controle preditivo. As vantagens dessas múltiplas aplicações, é considerada relevante, especialmente quando um processo é seriamente atingido por uma causa atribuível ou especial continuamente recorrente (ALVES, et al., 2012).

Os gráficos de controle com memória não são substitutivos aos gráficos de Shewhart, uma vez que são utilizados para detectar pequenos desvios, mas podem atuar como complemento aos mesmos, auxiliando na melhoria do desempenho do processo com a finalidade de atingirem níveis de estabilidade sempre melhores e portando melhor qualidade dos bens produzidos.

É importante salientar ainda que a implementação de qualquer gráfico de controle depende das especificidades de cada processo. Para tal, deve-se observar que é essencial selecionar adequadamente os seus parâmetros, uma vez que a eficiência destes gráficos depende em parte desta escolha.

\section{Referências}

ABBAS, N., RIAZ, M. and DOES, R.J.M.M. (2014). An EWMA-type control chart for monitoring the process mean using auxiliary information. Communication in Statistics -Theory Methods, v.43, p. 3485-3498, 2014.

ABBAS, N.; RIAZ, M. and DOES, R.J.M.M. Enhancing the performance of EWMA charts. Quality and Reliability Engineering International, 27(6):821-833, 2011.

ABBAS, N.; RIAZ, M. and DOES, R.J.M.M. Mixed exponentially weighted moving average-cumulative sum charts for process monitoring. Quality and Reliability Engineering International. 29(3):355-365, 2013.

ABBAS, N. and SAEED, U. Assorted control charts: An efficient statistical approach to monitor $\mathrm{pH}$ values in ecotoxicology lab, 33(6), p.1-20, Journal of Chemometrics, John Wiley \& Sons, 2019.

ALVES,C.C; HENNING,E.; KONRATH, A,C; WALTER, O.M.F.C e SAMOHYL, R.W. A estatística Média Móvel Exponencialmente Ponderada para o controle preditivo, monitoramento e ajuste de processos. Congresso Latino-Iberoamericano de Investigación Operativa (CLAIO) e Simpósio Brasileiro de Pesquisa Operacional (SBPO), Rio de Janeiro, Brazil, 2012.

ALVES,C.C; HENNING, E.; SAMOHYL, R.W.; AMARAL,C.E. e CRUZ, A.A. A utilização da função perda de Taguchi sob a ótica das regiões de máximo e mínimo ARL para otimizar os parâmetros estatísticos do gráfico de controle CUSUM. Inovação e Tecnologia na Engenharia de Produção, Cap. 2, pág. 35-56, Curitiba: Editora Contexto, 2013.

ALVES, C. C.; KONRATH, A. C.; HENNING, E. PALADINI, E. OLIVEIRA, T.A e OLIVEIRA, A. The Mixed CUSUMEWMA (MCE) control chart as a new alternative in the monitoring of a manufacturing process. Brazilian Journal of Operations \& Production Management, v.16, n. 1, p. 1-13, 2019. 
ALVES, C.C e SAMOHYL, R.W. A utilização dos gráficos de controle CUSUM para o monitoramento de processos industriais. XXIV Encontro Nacional de Engenharia de Produção - Florianópolis, SC, 2004.

ALVES,C.C; SAMOHYL, R.W. ZAGO,V.A e HENNING,E. Uma aplicação do gráfico de controle média móvel geralmente ponderada no processo de produção de papel. INOVAE - Journal of Engineering, Architecture and Technology Innovation, Vol.4(1), São Paulo, SP, 2016.

ASLAM, M.; RAO, G.S.; KHAN, N. and AL-ABBASI, F.A. EWMA Control Chart Using Repetitive Sampling for Monitoring Blood Glucose Levels in Type-II Diabetes Patients. Symmetry 2019, v.11 (1):57, 2019.

CASTAGLIOLA, P.; MARAVELAKIS, P.E. and FIGUEIREDO, F.O. The EWMA median chart with

estimated parameters. IIE Transactions, Issue 1 : Quality \& Reliability Engineering, v. 48, p 66-74, 2015.

COSTA, A.F.B.; EPPRECHT, E.K. e CARPINETTI, L.C.R. Controle estatístico de qualidade. $2^{\text {a }}$ edição, São Paulo: Atlas, 2005.

HAWKINS, D. M and WU, Q. The CUSUM and the EWMA Head-to-Head. Quality Engineering, v.26, p.215222, 2014.

HAWKINS, D.M. \& OLWELL, D.H Cumulative Sum Charts and Charting for Quality Improvement, Statistics for Engineering and Physical Science, Springer, 1998.

JARQUE, C. M. and BERA, A. K. A test for normality of observations and regression residuals. Int. Stat. Rev., Woodbury, v.55, p.163-172, 1987.

LOUZADA, F.; DINIZ, C.A.R.; FERREIRA, P. H.; FERREIRA, E. L. Controle Estatístico de Processos: Uma abordagem prática para cursos de Engenharia e Administração. 1aㅗ. ed., 282 pág., Rio, LTC Editora, 2013.

MACHADO, M.A.G. e COSTA, A.F.B. The double sampling and the EWMA charts based on the sample variances. International Journal of Production Economics 2008; 114(1):134-148, 2008.

MONTGOMERY, D.C. Introdução ao controle estatístico da qualidade. 7ạ edição, Rio de Janeiro: Editora LTC, University of Florida, USA, 2016.

NAZIR, H. Z.; ABBAS, N.; RIAZ, M. and DOES, R. J. M. M. A comparative study of memory-type control charts under normal and contaminated normal environments. Quality and Reliability Engineering International, v. 32, n. 4, p. 1347-1356, 2016.

QIL P. Introduction to statistical process control. 1st Ed., Chapman \& Hall/CRC, University of Florida,2014. RAJI, I.A.; ABBAS, N. and RIAZ, M. On designing a robust double exponentially weighted moving average control chart for process monitoring. Transactions of the Institute of Measurement and Control, v. 40(15) p.4253-4265, 2018.

RAJI, I. A. Robust dual memory control charting schemes. Dhahran, Saudi Arabia, 130 p., Dissertation (Master of Science in Applied Statistics) - King Fahd University of Petroleum \& Minerals, Dhahran, Saudi Arabia, 2015.

RYAN, T.P. Statistical methods for quality improvement 3rd Ed., John Wiley and Sons, Nova Jersey, 2011.

SANUSI, R.A.; MUKHERJEE, A. and XIE, M. A comparative study of some EWMA schemes for simultaneous monitoring of mean and variance of a Gaussian process. Computers \& Industrial Engineering, v. 135, p.426-439, 2019.

STEINER, S.H. EWMA control charts with time varying control limits and fast initial response. Journal of Quality Technology, 31(1):75-86, 1999.

WHEELER, D.J. Advanced Topics in Statistical Process Control: The Power of Shewhart's chart. SPC Press, Knoxville,1995.

ZAMAN, B.; RIAZ, M.; ABBAS, N. and DOES, R.J.M.M. Mixed Cumulative Sum-Exponentially Weighted Moving Average Control Charts: An Efficient Way of Monitoring Process Location. Quality and Reliability Engineering International, v. 31(8), pp. 1407-1421, 2014.

ZWETSLOOT, I.M. ; SCHOONHOVEN, M. and DOES, R. J. M. M. Robust point location estimators for the EWMA control chart. Quality Technology \& Quantitative Management, v.13 (1), 29-38, 2016. 